\title{
Exploitation of a $\beta$-lactamase reporter gene fusion in the carbapenem antibiotic production operon to study adaptive evolution in Erwinia carotovora
}

\author{
Correspondence \\ George P. C. Salmond \\ gpcs@mole.bio.cam.ac.uk
}

Received 6 October 2005

Revised 12 December 2005

Accepted 14 December 2005

\author{
Steven D. Bowden and George P. C. Salmond \\ Department of Biochemistry, University of Cambridge, Tennis Court Road, Cambridge \\ CB2 1OW, UK
}

\begin{abstract}
Erwinia carotovora subsp. carotovora strain ATTn10 produces the $\beta$-lactam antibiotic 1-carbapen2-em-3-carboxylic acid (carbapenem) by expressing the carABCDEFGH operon. Mutants exhibiting increased carbapenem gene transcription were positively selected using an engineered strain with a functional $\beta$-lactamase translational fusion in $\mathrm{carH}$, the last gene of the operon. However, spontaneous ampicillin-resistant mutants were isolated even when transcription of carH: : blaM was blocked by a strongly polar mutation in carE. The mechanism of resistance was shown to be due to cryptic IS10 elements transposing upstream of carH: : blaM, thereby providing new promoters enabling $\mathrm{carH}:$ : blaM transcription. Southern blots showed that IS10 was present in multicopy in ATTn10. In addition, a Tn10 genetic remnant was discovered. The results offer insights into the genetic archaeology of strain ATTn10 and highlight the powerful impacts of cryptic IS elements in bacterial adaptive evolution.
\end{abstract}

\section{INTRODUCTION}

Erwinia carotovora subsp. carotovora (Ecc) strain ATCC 39048 is a Gram-negative phytopathogenic member of the Enterobacteriaceae and a causal agent of potato soft rot. In our earlier development of genetic analysis tools for this strain, $\operatorname{Tn} 10$ was introduced by $\lambda:: \operatorname{Tn} 10$ mutagenesis into the genetically 'virgin' version of ATCC 39048, in the selection of a mutant defective in the endogenous DNA restriction system (McGowan et al., 1996). This restrictionless mutant strain was then subjected to fusaric acid eduction (Bochner et al., 1980) to yield the tetracycline (Tc)sensitive derivative strain ATTn10, which was still restrictionless (McGowan et al., 1996). ATTn10 has subsequently been studied as a model genetically tractable phytopathogen with which to understand the regulation of bacterial virulence factors involved in plant disease.

ATTn10 is known to express the carABCDEFGH gene operon, which enables production of the $\beta$-lactam antibiotic 1-carbapen-2-em-3-carboxylic acid (carbapenem, Car) (McGowan et al., 1996; reviewed by Coulthurst et al., 2005). The first three genes, $c a r A B C$, are essential for Car biosynthesis (McGowan et al., 1997). carDE are also involved in Car biosynthesis, but are not essential, because when they are disrupted by in-frame deletions, Car is still produced, though at a reduced level. carFG are involved in Car intrinsic resistance via an uncharacterized, novel

Abbreviations: Ap, ampicillin; Tc, tetracycline; Car, 1-carbapen-2-em-3carboxylic acid; Ecc, Erwinia carotovora subspecies carotovora. mechanism (McGowan et al., 1997). The presence of carFG does not confer cross-resistance to related $\beta$-lactam antibiotics such as imipenem (McGowan et al., 1997). CarF and CarG both contain predicted signal peptides that direct their export to the periplasm (McGowan et al., 1997). The carH gene product has no known function, but is also exported to the periplasm (McGowan et al., 1997).

It has been shown recently that the ATTn10 carABCDEFGH gene cluster contains two promoters (McGowan et al., 2005). The first promoter (P1) is located immediately upstream of carA. Transcription from $\mathrm{P} 1$ is increased by the presence of the quorum-sensing signal molecule, $\mathrm{N}$-(3-oxohexanoyl)-Lhomoserine lactone (OHHL), produced by the CarI enzyme (McGowan et al., 1996). The OHHL signal molecule interacts with CarR with a dissociation constant of $1 \cdot 8 \mu \mathrm{M}$ (Welch et al., 2000). It is thought that the CarR: OHHL complex activates transcription of the carABCDEFGH operon, and that this leads to production of Car (McGowan et al., 1997). The second promoter (P2) is located within carD and is not regulated by quorum sensing (McGowan et al., 2005). P2 is a weak promoter that allows low levels of expression of a carG : : lacZ transcriptional fusion (McGowan et al., 2005). P2 is presumed to maintain auto-resistance to leaky production of Car by an individual cell or by its neighbours.

The aim of this research was to create and exploit a strain with a $\beta$-lactamase translational fusion in the $\mathrm{carH}$ gene to enable the positive selection of mutants exhibiting increased transcription of the carABCDEFGH operon. We presumed that these spontaneous mutants would have point mutations 
within the $\mathrm{P} 1$ and $\mathrm{P} 2$ promoters that would lead to increased expression of the carABCDEFGH genes. In addition, we predicted that we might isolate a class of mutants with extra-operonic mutations defining new genes involved in carbapenem operon regulation. However, our results were surprisingly unexpected, and led to the discovery that $\operatorname{Tn} 10$ had not been entirely lost from the ATTn10 genome, highlighting the powerful role of mobile genetic elements in the adaptive evolution of bacteria that are challenged by a potentially lethal chemical selection pressure.

\section{METHODS}

Bacterial strains, plasmids and growth conditions. All $E c c$ and Escherichia coli strains, bacteriophage and plasmids used in this study are listed in Table 1 . Strains were routinely grown on
Luria-Bertani broth agar (LBA) (Miller, 1972), at $30^{\circ} \mathrm{C}$ for $E c c$ strains and $37^{\circ} \mathrm{C}$ for E. coli strains, supplemented with antibiotics, as necessary. Liquid cultures of Ecc were grown in sterile conical flasks containing one-tenth volume Luria-Bertani broth (LB) at $30^{\circ} \mathrm{C}$ at 300 r.p.m. in shaking water baths.

DNA manipulations and sequencing. All molecular biological techniques, unless stated otherwise, were performed by standard procedures (Sambrook et al., 1989). Restriction enzymes and T4 DNA ligase were purchased from NEB and were used according to the manufacturer's instructions. Oligonucleotide primers were obtained from Sigma Genosys and are listed in Table 2. DNA sequencing was performed by the DNA sequencing facility, University of Cambridge. Nucleotide sequence data were analysed using the GCG package (Genetics Computer Group, University of Wisconsin); sequences were compared to protein and nucleotide databases using the BLAST suite of programs (Altschul et al., 1990) at GenBank (http://www.ncbi.nlm.nih.gov/BLAST/).

Table 1. Strains, plasmids and phages used in this study

\begin{tabular}{|c|c|c|}
\hline Strain & Relevant genotype or description & Reference or source \\
\hline \multicolumn{3}{|l|}{ E. coli } \\
\hline CC118ipir & $\begin{array}{l}\text { araD139 } \Delta(\text { ara leu }) 7697 \Delta l a c X 74 \text { phoA20 galE galK thi rpsE rpoB } \\
\text { araEam recA1 ( } \lambda \text { pir })\end{array}$ & Herrero et al. (1990) \\
\hline HH26 (pNJ5000) & E. coli helper strain used in marker exchange & Grinter (1983) \\
\hline ESS & $\beta$-Lactam super-sensitive strain used in Car bioassays & Bainton et al. (1992) \\
\hline \multicolumn{3}{|l|}{$E c c$} \\
\hline ATCC 39048 & Original 'virgin' $\mathrm{Car}^{+}$strain & Laboratory stock \\
\hline ATTn10 & $\begin{array}{l}\text { Restrictionless } \mathrm{Tc}^{\mathrm{S}} \text { derivative of a Tn } 10 \text {-containing ATCC } 39048 \\
\text { (made by fusaric acid curing) }\end{array}$ & McGowan et al. (1996) \\
\hline SBE1 & carH: : blaM derivative of ATTn10 & This study \\
\hline SM11 & $\Delta c a r B$ derivative of ATTn10; $\mathrm{Car}^{-}$ & McGowan et al. (1997) \\
\hline SBE5 & carH: : blaM derivative of SM11 & This study \\
\hline SBE7 & $\Delta c a r B, \operatorname{carE}:: \Omega, \operatorname{carH}::$ blaM derivative of SBE5 & This study \\
\hline SBE9 & $\Delta \operatorname{carB}, \operatorname{carE}:: \Omega, \operatorname{carH}::$ blaM derivative of ATCC 39048 & This study \\
\hline SBE7 5.1 & Spontaneous ampicillin-resistant mutant derived from SBE7 & This study \\
\hline SBE7 5.2 & Spontaneous ampicillin-resistant mutant derived from SBE7 & This study \\
\hline SBE7 5.3 & Spontaneous ampicillin-resistant mutant derived from SBE7 & This study \\
\hline SBE7 5.4 & Spontaneous ampicillin-resistant mutant derived from SBE7 & This study \\
\hline SBE7 5.5 & Spontaneous ampicillin-resistant mutant derived from SBE7 & This study \\
\hline SBE7 5.6 & Spontaneous ampicillin-resistant mutant derived from SBE7 & This study \\
\hline SBE7 5.7 & Spontaneous ampicillin-resistant mutant derived from SBE7 & This study \\
\hline SBE7 5.8 & Spontaneous ampicillin-resistant mutant derived from SBE7 & This study \\
\hline SBE7 5.9 & Spontaneous ampicillin-resistant mutant derived from SBE7 & This study \\
\hline SBE7 5.10 & Spontaneous ampicillin-resistant mutant derived from SBE7 & This study \\
\hline \multicolumn{3}{|l|}{ Plasmid } \\
\hline pKNG101 & $\operatorname{sacB}$, oriR6K, $\mathrm{Sm}^{\mathrm{R}}$ & Kaniga et al. (1991) \\
\hline pSMG45 & 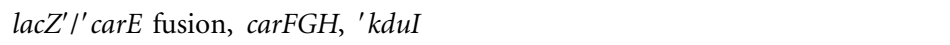 & McGowan et al. (1997) \\
\hline pSMG45HBlaMCAA + & pSMG45 plasmid with EZ TnBlaM carH translational fusion (see Methods) & This study \\
\hline pSMG45HBlaMTAA + & pSMG45HBlaMCAA + with stop codon corrected to TAA (see Methods) & This study \\
\hline pSMG45HBlaMTAA- & $\begin{array}{l}\text { SmaI/SnaBI-digested pSMG45HBlaMTAA }+6 \cdot 2 \mathrm{~kb} \text { band self-ligated } \\
\text { to remove oriR6K }\end{array}$ & This study \\
\hline pKNG101HBlaMTAA- & $\begin{array}{l}\text { BglII-SpeI } 2 \cdot 4 \mathrm{~kb} \text { pSMG45HBlaMTAA - band ligated into BamHI- } \\
\text { XbaI } 6 \cdot 5 \mathrm{~kb} \text { pKNG101 band }\end{array}$ & This study \\
\hline pSMG112 & $\operatorname{carD}, \operatorname{car} E:: \Omega, \operatorname{carF}, \operatorname{car} G^{\prime}$ cloned into pKNG101 & McGowan et al. (2005) \\
\hline \multicolumn{3}{|l|}{ Bacteriophage } \\
\hline$\phi \mathrm{KP}$ & Generalized Ecc transducing phage & Toth et al. (1993) \\
\hline
\end{tabular}


Table 2. Primers used in this study

\begin{tabular}{|ll|}
\hline Primer & \multicolumn{1}{c|}{ Sequence $\left(\mathbf{5}^{\prime} \mathbf{- 3}^{\prime} \mathbf{)}\right.$} \\
\hline CarF5' & CGCTAATTGTTGGACTGTGCTG \\
BlaRP1 & CCAGCGTTTCTGGGTGATCGATGAT \\
CarHF1 & CATGAATTCGGTAACACAAGATACCTACC \\
CarHB1 & CATGGATCCTTAGTAATCCAGCTCTACCG \\
INBlaM3' & CATGGTACCTTACCAATGCTTAATCAGTGA \\
IS105'2 & ACAAGATGTGCGAACTCGAT \\
IS103' & TCCCCAAAGCGTAACCATGT \\
jemC1 & AGCATAACCTTTTTCCGTGATGGT \\
jemC2 & GATCTATGATTCCCTTTGTCAACAGCA \\
Sp2 & GCCTTGATGTTACCCGAGAGC \\
\hline
\end{tabular}

Engineering of carH::blaM translational fusion plasmids. The plasmid pSMG45 was mutagenized in vitro using the EZ TnBlaM kit (Epicentre) as described in the manufacturer's manual. Briefly, $1 \mu \mathrm{l}$ reaction mixture was used to transform electrocompetent CC118גpir cells, which were plated onto LBA supplemented with $5 \mu \mathrm{g}$ ampicillin (Ap) $\mathrm{ml}^{-1}$ and incubated overnight at $37^{\circ} \mathrm{C}$. Resistant transformant colonies were screened by colony PCR using the primers CarF5' and BlaRP1. One colony was identified as carrying an insertion in $c a r H$. Sequencing of the $c a r H$ gene using primers CarHF1 and CarHB1 confirmed that the EZ TnBlaM had inserted into the $c a r H$ gene at bp 7713 as defined by the car $A-H$ gene cluster accession number U17224. This insertion was predicted to yield a functional translational fusion of the $\mathrm{carH}$ and blaM genes.

Unfortunately, sequencing revealed that bp 823 of the original EZ TnBlaM transposon DNA was a cytosine residue rather than the documented thymine residue listed in the manufacturer's manual. This single base pair alteration had the effect of altering the blaM TAA stop codon to a CAA codon encoding glutamine. Furthermore, this base pair alteration extended the ORF of the blaM gene by an additional 23 codons.

To correct the T-C base pair alteration, a PCR strategy was devised. Briefly, primers CarF5' and INBlaM3' were used to amplify carFG, carH' : blaM from pSMG45HBlaMCAA + using the Hi Fi PCR kit supplied by Roche. The PCR product was digested with SpeI and $K p n \mathrm{I}$ and cloned into the $5.0 \mathrm{~kb}$ pSMG45HBlaMCAA + SpeI/KpnI band to generate the plasmid pSMG45HBlaMTAA +. CC118 $\lambda$ pir (pSMG45HBlaMTAA +) grown on LBA had a MIC $>500 \mu \mathrm{g}$ Ap $\mathrm{ml}^{-1}$, whilst CC118ipir (pSMG45HBlaMCAA + ) had an MIC of $<50 \mu \mathrm{g} \mathrm{Ap} \mathrm{ml}^{-1}$, confirming that the altered stop codon was dramatically influencing $\mathrm{CarH} \phi \mathrm{BlaM} \beta$-lactamase activity in $E$. coli (data not shown).

Marker exchange. Engineering of carE:: $\Omega$ and $c a r H::$ blaM was carried out using the pKNG101-derived plasmids pSMG112 and pKNG101HBlaMTAA-, respectively, as described previously (Kaniga et al., 1991). To avoid selecting for unwanted mutations during the marker exchange process that could lead to enhanced $\beta$ lactamase activity from the carH: blaM mutation, Ap was not used to select for colonies containing the carH:: blaM marker. Instead, sucrose-resistant, streptomycin $(\mathrm{Sm})$-sensitive colonies were screened by patching onto LBA and LBA plus $5 \mu \mathrm{g} \mathrm{Ap} \mathrm{ml}^{-1}$. Any colonies that were able to grow on the LBA plus $5 \mu \mathrm{g} \mathrm{Ap} \mathrm{ml}^{-1}$ plates were then picked from the LBA-only plate, and the presence of the carH: : blaM marker was confirmed by PCR analysis.

Carbapenem plate assays. Carbapenem production assays on overnight cultures of Ecc samples were carried out as described previously (McGowan et al., 1996), using the $\beta$-lactam sensor strain
ESS. The only alteration was that, in some experiments, freshly prepared stock solutions of clavulanate were added to the LBA overlay to the desired final concentration to inhibit carH::blaM $\beta$-lactamase activity (Reading \& Cole, 1977).

Nitrocefin-based $\boldsymbol{\beta}$-lactamase assays. Expression of carH: : blaM throughout growth was assayed using the chromogenic substrate nitrocefin (GlaxoSmithKline). Aliquots $(1 \mathrm{ml})$ were extracted from liquid cultures of $E c c$ and resuspended in phosphate buffer, $\mathrm{pH} 7 \cdot 0$. The samples were then sonicated on ice for a total time of $3 \mathrm{~min}$ and $45 \mathrm{~s}$, composed of eight $15 \mathrm{~s}$ periods of sonication with $15 \mathrm{~s}$ gaps between them. Cell debris was pelleted using a benchtop centrifuge at 13000 r.p.m. for $5 \mathrm{~min}$. To $800 \mu \mathrm{l}$ of the sample incubated at $30^{\circ} \mathrm{C}$, $20 \mu \mathrm{l}$ of $4 \mathrm{mg}$ nitrocefin $\mathrm{ml}^{-1}$ stock solution was added, and the change in $\mathrm{OD}_{550}$ was recorded in a spectrophotometer over time and recorded relative to the $\mathrm{OD}_{600}$ of the culture at each time-point.

Southern blotting using DIG-labelled probes. Southern blotting experiments were carried out using standard procedures (Sambrook et al., 1989). DIG-labelled probes hybridizing to IS10 and jem $C$ were created by PCR using the DIG-labelled dNTP mix supplied by Roche. The IS10 probe was PCR-amplified using primers IS105' 2 and IS103'. DIG-labelled probes were detected using $\mathrm{FAb}$ fragments supplied by Roche. Peroxidase activity was detected using the CDP-star kit supplied by Roche.

\section{RESULTS AND DISCUSSION}

\section{CarH $\phi$ BlaM is functionally expressed in Ecc throughout growth}

A carH: : blaM translational fusion was constructed in the ATTn10 carA-H operon by marker exchange to generate
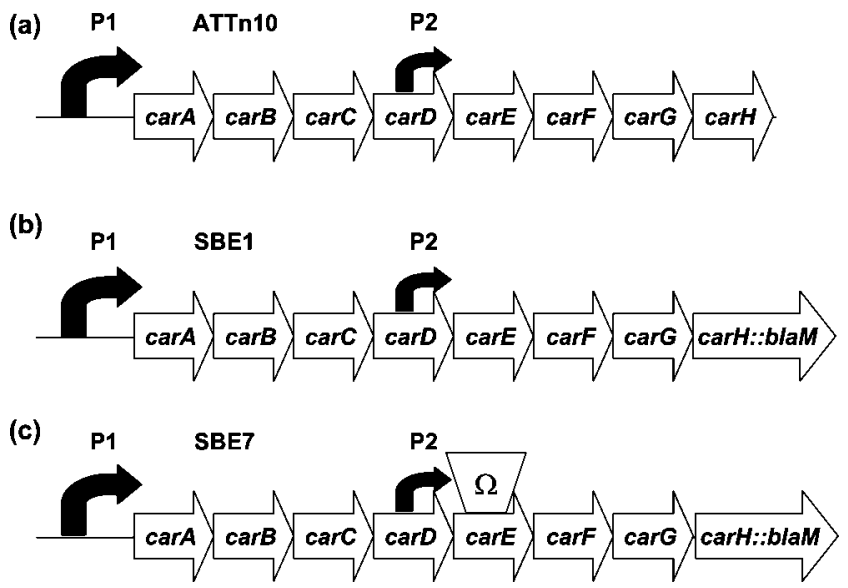

Fig. 1. (a) Diagrammatic representation of the carA-H cluster in ATTn10. The P1 promoter enables transcription of carA-H and is regulated by the CarlR quorum-sensing system (McGowan et al. 1996). The P2 promoter is located within carD and enables weak expression of CarEFGH. (b) The carA$H$ cluster in SBE1 has a $\beta$-lactamase translational fusion in the carH gene. (c) SBE7 has an identical carA-H gene cluster to that of SBE1, except that an $\Omega$ interposon in carE prevents expression of CarF, CarG and CarH $\phi$ BlaM from the $\mathrm{P} 1$ and $\mathrm{P} 2$ promoters. 


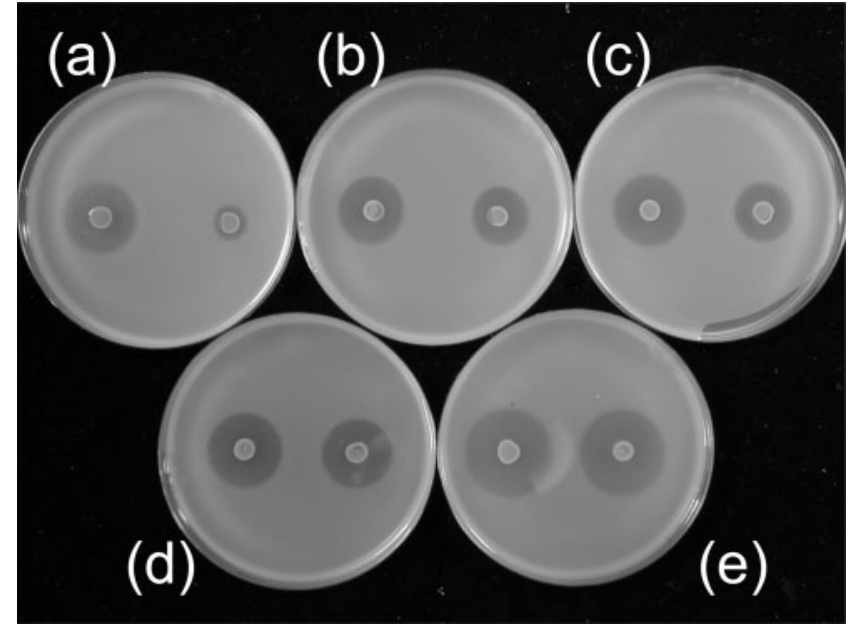

Fig. 2. Clavulanate inhibits carbapenem degradation by CarH $\phi$ BlaM $\beta$-lactamase activity. Spots of ATTn10 (left hand side of each plate) and SBE1 (right hand side of each plate) were added to $0.7 \%$ Luria broth top agar, which had been seeded with the $\beta$-lactam sensor strain ESS, containing increasing concentrations of clavulanate: (a) $0 \mu \mathrm{g}$ clavulanate $\mathrm{ml}^{-1}$, (b) $5 \mu \mathrm{g}$ clavulanate $\mathrm{ml}^{-1}$, (c) $10 \mu \mathrm{g}$ clavulanate $\mathrm{ml}^{-1}$, (d) $25 \mu \mathrm{g}$ clavulanate $\mathrm{ml}^{-1}$ and (e) $50 \mu \mathrm{g}$ clavulanate $\mathrm{ml}^{-1}$, and incubated overnight at $30^{\circ} \mathrm{C}$. The zones of clearing are proportional to the levels of carbapenem secreted by ATTn10 and SBE1.

strain SBE1 (Fig. 1b). To test whether the $\mathrm{CarH} \phi \mathrm{BlaM}$ protein was functional in vivo, ATTn10 and SBE1 Car production was assessed by spotting cultures onto ESS overlays supplemented with clavulanate (Fig. 2). Clavulanate was used to inhibit the $\beta$-lactamase activity (Reading \& Cole, 1977) conferred by CarH $\phi$ BlaM. In the absence of clavulanate, the zone of clearing on the ESS lawn around SBE1 was reduced compared to that of ATTn10 (Fig. 2a). However, addition of clavulanate to the ESS overlays restored the size of the Car haloes around SBE1 to the same as that of ATTn10 (Fig. 2b-e). These results confirmed that i) SBE1 was producing the same levels of Car as that of ATTn10, and ii) the $\mathrm{CarH} \phi \mathrm{BlaM} \beta$-lactamase was functional, as it could hydrolyse Car unless excess clavulanate was present as a competitive inhibitor.
CarH $\phi$ BlaM expression was detected throughout growth by measuring $\beta$-lactamase activity (Fig. 3). The level of $\beta$ lactamase activity in SBE1 increased dramatically during mid-exponential growth, as predicted, due to car $A-H$ transcription via quorum sensing. The level of $\beta$-lactamase activity from SBE1 began to decline again once the cells were in stationary phase, suggesting that the $\mathrm{CarH} \phi \mathrm{BlaM}$ hybrid was turned over in vivo, presumably because of proteolysis. Previous studies have shown that $\beta$-galactosidase also declines during stationary phase (McGowan et al. 2005). It seems likely that both $\mathrm{CarH} \phi \mathrm{BlaM}$ and $\beta$-galactosidase are inactivated by proteases that are induced, or are most active, during the stationary phase of growth.

A third strain (SBE7, Fig. 1c) was constructed, in which transcription of $c a r H:$ : blaM was disrupted. SBE7 $c a r H:$ : blaM transcription from both of the known carA$H$ promoters was blocked by the presence of the strongly polar omega interposon sequence (Prentki \& Krisch, 1984) in carE (Figs $1 \mathrm{c}$ and 3). Consistent with our previous data on transcription of the operon (McGowan et al., 2005), transcription of carH:: blaM was switched off in strain SBE7.

\section{The carH:: blaM fusion strain can give rise to ampicillin-resistant mutants, despite the interposon insertion}

The survival frequency of ATTn10 and SBE1 in response to increasing concentrations of Ap is recorded in Fig. 4. As a negative control we also included Ecc strain SBE7, which cannot transcribe the carH::blaM gene because of the carE: $: \Omega$ mutation, as described above. Surprisingly, SBE7 had an e.o.p. on LBA supplemented with $5 \mu \mathrm{g} \mathrm{Ap} \mathrm{ml} \mathrm{m}^{-1}$ of $1 \times 10^{-5}$, which was significant, because ATTn10 could not grow on this medium. Ecc growth on LBA supplemented with $25 \mu \mathrm{g} \mathrm{Ap} \mathrm{ml}^{-1}$ was undetectable after $24 \mathrm{~h}$ at $30^{\circ} \mathrm{C}$, with the exception of SBE1, which had an e.o.p. of $1 \times 10^{-7}$ relative to that in the absence of Ap.

The e.o.p. values of SBE1 and SBE7 when grown on LBA supplemented with $5 \mu \mathrm{g} \mathrm{Ap} \mathrm{m}{ }^{-1}$ were $1 \times 10^{-4}$ and $1 \times$ $10^{-5}$, respectively. Because transcription of carH:: blaM in SBE7 cannot occur from either of the carA-H promoters because of the polar carE:: $\Omega$ mutation, SBE7

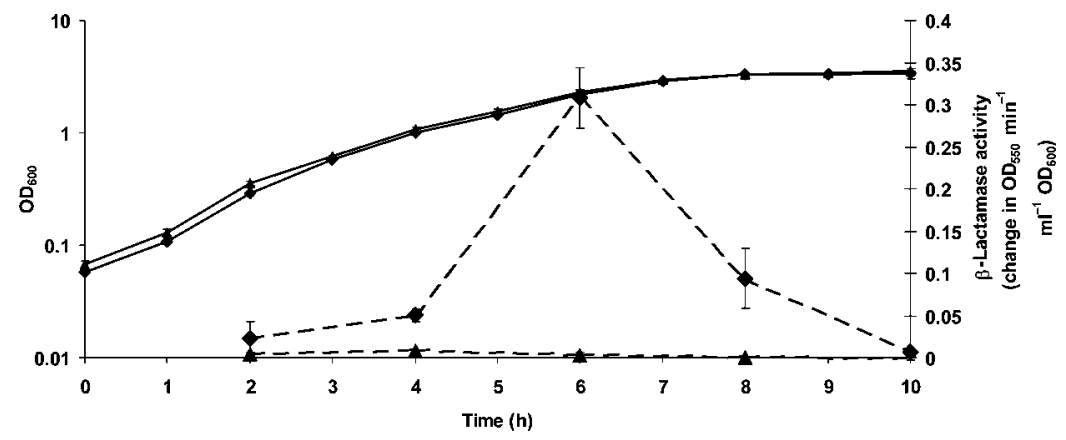

Fig. 3. Detection of $\beta$-lactamase activity throughout growth using the chromogenic cephalosporin nitrocefin. The following are shown: $\log \mathrm{OD}_{600}$ of SBE1 (diamonds, solid line) and SBE7 (triangles, solid line) grown at $30^{\circ} \mathrm{C}$ in Luria broth at 300 r.p.m. in shaking water baths; $\beta$-lactamase activity of SBE1 (diamonds, broken line) and SBE7 (triangles, broken line) throughout growth. 


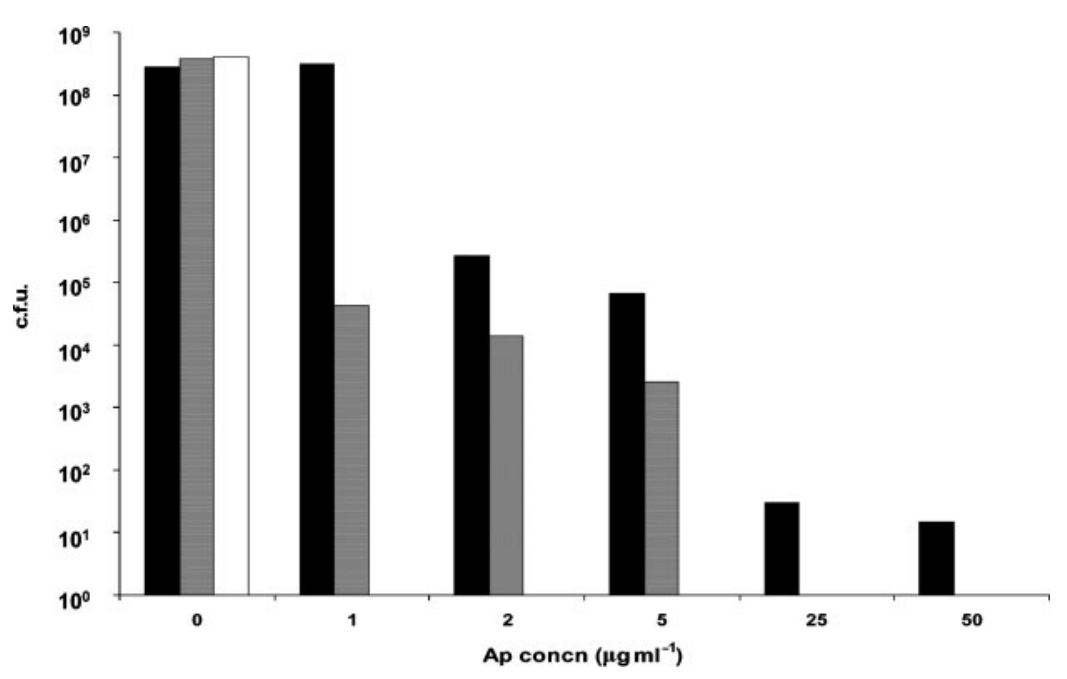

Fig. 4. Survival efficiency of SBE1 (black bars), SBE7 (grey bars) and ATTn10 (white bars) in response to different Ap concentrations.

$\mathrm{Ap}^{\mathrm{R}}$ colonies could only arise if the $\mathrm{carH}:$ : blaM fusion was transcribed from a new promoter.

\section{Expression of CarH $\phi$ BlaM can occur spontaneously in SBE7 because of the action of mobile DNA providing functional promoters}

To determine the nature of the SBE7 Ap ${ }^{\mathrm{R}}$ mutants, 10 of the colonies (SBE7 5.1-5.10) that grew on $5 \mu \mathrm{g} \mathrm{Ap} \mathrm{ml}^{-1}$ were selected at random for further investigation. The most likely explanation for $\mathrm{Ap}^{\mathrm{R}}$ in SBE7 5.1-5.10 was that a genetic alteration (presumably insertions or deletions) had occurred immediately upstream of carH::blaM. Therefore, the region between $\operatorname{carH}:: b l a M$ and $\operatorname{car} E:: \Omega$ in these mutants was amplified by PCR analysis using primers Sp2 and BlaRP1 (Fig. 5a). It was clear that, in the Ap ${ }^{\mathrm{R}}$ SBE7 mutants, the size of the $1871 \mathrm{bp}$ region (Fig. 5a, lane F) between carH: blaM and carE:: $\Omega$ had increased. In most cases, the increase in size was approximately $1.3 \mathrm{~kb}$. In the case of SBE7 5.5, the increase in size was approximately $1.8 \mathrm{~kb}$ (Fig. 5a, lane E). Sequencing of four of the PCR products from SBE7 5.1 to 5.4 revealed that IS10R from the transposon $\operatorname{Tn} 10$ had inserted in the region between carH:: blaM and carE:: $\Omega$ (Fig. 5b). The site of the IS10 insertion in strains SBE7 5.1, 5.3 and 5.4 was identical in each case, suggesting that they could be siblings. In all cases, the IS10R $\mathrm{P}_{\text {out }}$ promoter was orientated in the correct direction to enable transcription of the downstream carH: : blaM gene (Fig. 5c). Previous data has shown that both IS10 elements possess a fairly strong $\mathrm{P}_{\text {out }}$ promoter (Simons et al., 1983) that is capable of allowing expression of adjacent ORFs (Ciampi et al., 1982). It seems that, due to these random insertions, the $\mathrm{P}_{\text {out }}$ promoter from IS10 has enabled SBE7 5.1-5.10 to drive transcription of the otherwise cryptic carH: : blaM fusion.

Our results suggest that ATTn10 provides a useful model to study the adaptive evolution processes seen in natural isolates that possess IS10R. As an example, earlier research by other groups has shown that IS elements can induce antibiotic resistance in E. coli (Jellen-Ritter \& Kern, 2001; Kobayashi et al., 2001) and Salmonella enterica (Olliver et al., 2005). In all three of these studies, IS elements were shown to provide mechanisms of broad-spectrum antibiotic resistance by providing promoters that are capable of expressing the multidrug resistance pump AcrEF. Such experiments show that IS elements can play a crucial role in the evolution of antibiotic resistance in clinical isolates.

\section{Transposon Tn10 has been extensively degraded in the ATTn10 genome}

Mutant SBE7 5.5 was particularly interesting, because the PCR product from this mutant was enlarged by approximately $1.8 \mathrm{~kb}$ instead of $1.3 \mathrm{~kb}$ (Fig. 5a, lane E). Sequencing of the enlarged PCR product showed that this mutant contained genetic remnants of $\operatorname{Tn} 10$ orientated such that the IS10L end was closest to carH::blaM. In SBE7 5.5, transcription of carH: :blaM appears to be from the $\mathrm{P}_{\text {out }}$ promoter of IS10L, as this promoter is in the correct orientation. The Tn10 sequence in this IS element was severely degraded, however, by the presence of two large deletions in its sequence (Fig. 6). Both deletions are defined by the Tn 10 sequence accession number AF162223 (Chalmers et al., 2000), and are as follows: deletion 1 removes bp 340-4426, which includes most of IS10L and ends in the $5^{\prime}$ end of jem $C$, removing jem $A B$ in the process; deletion 2 removes bp 4698-7818. Deletion 2 removes the $3^{\prime}$ end of the jem $C$ gene and the entire tetRACD gene cluster, and ends precisely at the point at which the IS10R inverted repeat sequence begins. This $1939 \mathrm{bp} \operatorname{Tn} 10$ genetic remnant will from now on be referred to as $\operatorname{Tn} 10 \Delta$.

The process leading to the generation of deletion 1 is not clear, but may involve strand slippage mechanisms (Farabaugh et al., 1978; Albertini et al., 1982; Singer \& Westlye, 1988; Marvo et al., 1983; Uematsu et al., 1999) during DNA replication or repair, because it is flanked at the 
(a)

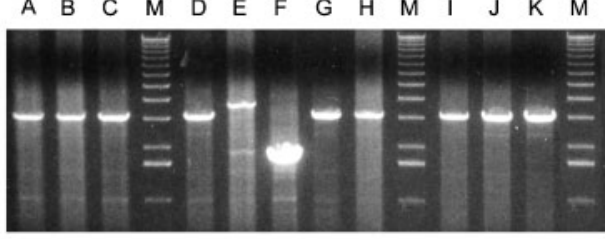

(c) (b)

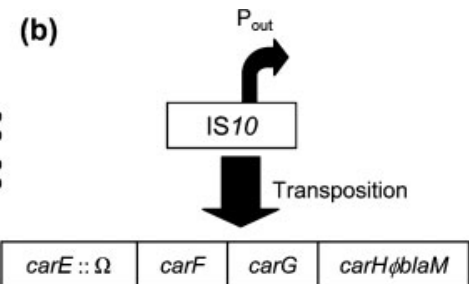

carF

$\longrightarrow$ $\leftarrow 4072$ bp $\leftarrow 3054 \mathrm{bp}$ $\leftarrow 2036 \mathrm{bp}$

6121 tgttctgtgc cgctctctaa cctcgaaatt gaacctgtat cgtcatgletg aagaaacgc 6181 taattgttgg actgtgctgt actttccccc tcttctccgc tcaagcggtg aatactgttc 6241 ctgacgaggt ggttgtcaaa ggtggcaatt tctatgttgg atcggtcttc ggctcggaag 6301 actatgctgc tcatgccaac acgtctatcg cgtcttttgc catcacgaaa acggaaatta 6361 cctatcggca gtatcatgcg ttgcaagaat gggcggacac acacggttat gagcttagtg 6421 gcggctgcaa tggcgcgact ttcgaagatt gcttaccgcc tgaacaggat aacagtcttc 6481 atcctgtcac gaatgtatct tggtgggatg cagtgatttt tgccaatgtg ctcagtgaac 6541 ggcagcagtt gcagccetat tacctcacga ttgacggtaa gacgetgaaa cgcgtgccgg 6601 aggacgataa cgataactc atacgcgaga acccgcaggc tttggggtat cgcttgccga 6661 cgctggcgga atggcaagtg gcagccagag gcggaaagaa ggggetggcg caggggacgt 6721 acggacagcg ctatgccggt agcgaacagc cggacagtgt ggcgcatttt ccttctgact 6781 ctcaatcatt cggcacggtg cetgtgacct cgaagcgccc caatgctctg gGGCTTTACG 6841 atatgagtgg caatgtatcc gagtggetga atgagtctta tgcggtggaa ggcggcaaaa 6901 ccatgtacta cttttgcggc ggaagttatc tggaacgcac gcacagtctt gctagctgcg 6961 atctgcatac gcceggtttt ttcaTGCCGG ATAttggttt tcgactagta aggacacttg 7021 atggtcaata agttcgtagg gtggctggcg ctctgtgcca ttagcaacac ggcTGCTGCA 7081 CTctctcctg tgacattgaa agacggcatc aatcggetgg atctcaatca gggcggcggg 7141 aacgattatg tcgttgttgc ccaatttgac aacaatacct cgcatcctaa tctcgggatg 7201 accttttttg tccgccgtcc agacggtggg catagcatta tgccggtagc caacagcaat 7261 acgtttacet ggttcgacta tcgectgtcc getgcggegg attttctggt gcaggacaat 7321 cggctattcc tgtctggaaa gcattacttt ctggtgacgg cacggaagca gggtgaaaac 7381 gtctttgatc ccacaaagt cgttttaaca atttacgact ttaaagcctc acgggacgat 7441 cccggtgtac cactctatga atggtcggag cgcaagcgtg tggtaacaca agatacctac 7501 caatcggtcg atgaggccta caaggaagtg aatgaggcga tgctggcaaa atgaaatat

Fig. 5. (a) Colony PCR analysis of the following strains using primers BlaRP1 and SP2: A, SBE7 5.1; B, SBE7 5.2; C, SBE7 5.3; D, SBE7 5.4; E, SBE7 5.5; F, SBE7; G, SBE7 5.6; H, SBE7 5.7; I, SBE7 5.8; J, SBE7 5.9; K, SBE7 5.10. The lanes designated $M$ are GibcoBRL Life Sciences 1 kb ladders. (b) Diagrammatic representation of IS10R transposition upstream of carH:: blaM but downstream of carE:: $\Omega$, leading to the IS1OR $\mathrm{P}_{\text {out }}$ promoter becoming correctly orientated to enable transcription of carH: : blaM. (c) The DNA sequence of carF and carG from GenBank accession number U17224. Boxed 'atg' sequences indicate the start codons of carF and carG. The stop codons for carF and carG are underlined and in italics. The bold sequences in upper-case type represent the sites of the IS10 insertions in SBE7 5.1-5.5. Three of the mutants, SBE7 $5.1,5.3$ and 5.4, had IS10 insertions that duplicated bp 7074-7082. One of the mutants, SBE7 5.2, had an insertion that duplicated bp 6985-6993. The last mutant, SBE7 5.5, had an enlarged IS10 insertion that duplicated bp 6832-6840.

termini by an imperfect 7 bp repeat sequence $\mathrm{G}(\mathrm{C} / \mathrm{A}) \mathrm{T}(\mathrm{G} /$ C)TAT. Alternatively, processes other than strand slippage (Balbinder, 2001) may be responsible for deletion 1 .

Deletion 2 terminates with a 5 bp imperfect direct repeat, ATGC(T/A), and therefore may have formed by a strand misalignment mechanism (Farabaugh et al., 1978; Albertini et al., 1982; Singer \& Westlye, 1988; Marvo et al., 1983; Uematsu et al., 1999). However, the direct transition of the IS $10 \mathrm{R}$ inverted repeat sequence that defines the boundaries of the mobile genetic element to the site of a deletion is characteristic of an IS10 adjacent deletion, as described elsewhere (Roberts et al., 1991; Chalmers \& Kleckner, 1996). It seems very likely that deletion 2 has been generated by an IS10-mediated adjacent deletion. Two lines of evidence support this hypothesis: i) deletion 2 is immediately flanked at the $3^{\prime}$ end by the IS 10 inside end, and ii) deletion 2 is immediately flanked at the $5^{\prime}$ end by a $5^{\prime}$-GGTTATGCT- $3^{\prime}$ sequence which closely matches the $5^{\prime}$-NGCTNAGCN-3'
IS 10 transposition consensus sequence (Halling \& Kleckner, 1982).

The $\mathrm{Tc}^{\mathrm{S}}$ phenotype of the ATTn10 strain can be explained by the presence of deletion 2. This is because deletion 2 has led to the complete loss of the tetRACD gene cluster from Tn 10. Furthermore, a Southern blot to probe for jem $C$ in ATTn10 genomic DNA cut with BsiEI, which cuts either end of Tn 10 , generated only one hybridizing band that corresponded to the size expected for Tn $10 \Delta$, confirming that the tetRACD and jemAB genes are absent in the ATTn10 genome (data not shown). Deletion 2 would offer a selective advantage when Tn10-containing strains were exposed to fusaric acid because it removes the tet $A$ gene that encodes the TetA efflux pump which confers fusaric acid sensitivity. An example of how fusaric acid treatment can lead to the generation of hybrid IS elements in strains containing Tn 10 has been documented in E. coli (Bogosian et al., 1993). The IS10R fusion in the strain under study generated a 1329 bp element 
(a)

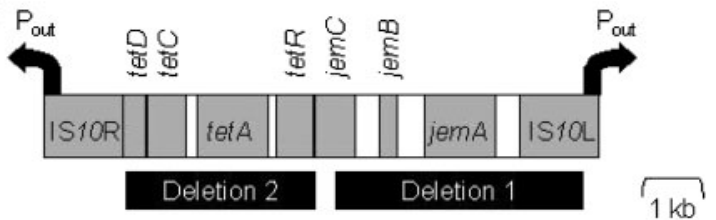

(b)

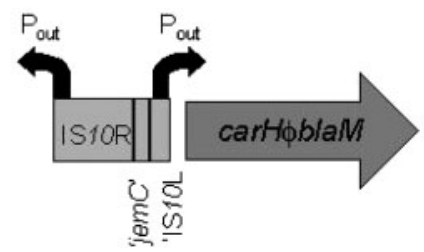

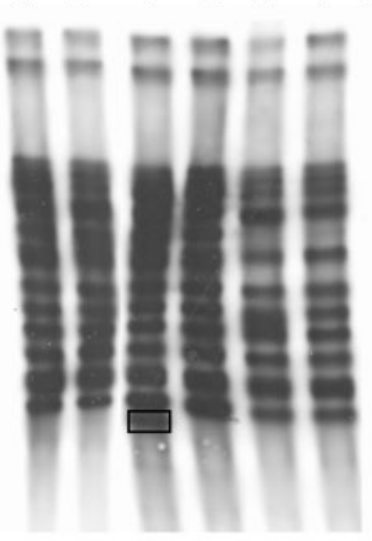

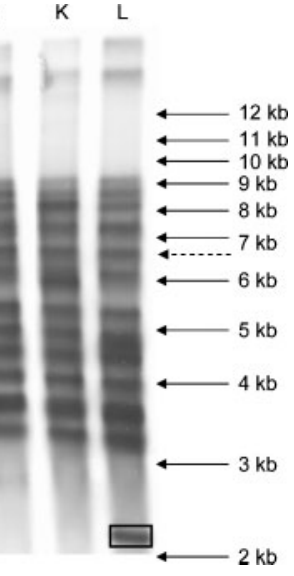

$2 \mathrm{~kb}$

Fig. 6. (a) Diagrammatic representation of the regions of $\operatorname{Tn} 10$ that have been deleted in Tn104. Genes and IS10 elements are represented as grey rectangles; deletions are represented as black rectangles. (b) Representation of $\mathrm{Tn} 10 \Delta$ insertion upstream of $\mathrm{carH}:$ : blaM in Ecc strain SBE7 5.5. Transcription of carH: :blaM is likely to occur from the $\mathrm{P}_{\text {out }}$ promoter of the IS10L fragment within Tn104 which is represented by a black bent arrow. All genes are drawn approximately to scale, with the exception of carH: : blaM.

(designated IS10L/R1) that contained sequence from IS10R as well as sequence from IS $10 \mathrm{~L}$. The Tn $10 \Delta$ element isolated in strain SBE7 5.5 is a new example of how the strong selection pressure exacted by fusaric acid treatment of $\operatorname{Tn} 10-$ containing strains can encourage the evolution of novel, hybrid IS elements.

\section{ATTn10 contains multiple copies of IS10}

Genomic DNA from SBE7, SBE9 and SBE7 5.1-5.10 was analysed by Southern blot experiments using DIG-labelled probes to the transposase gene of IS10 (Fig. 7). No hybridizing bands were detected in SBE9 DNA. SBE9 is not derived from ATTn10, and therefore has never been exposed to Tn10. However, it was clear from the SBE7 sample, which does derive from ATTn10, that there were at least 15 independent hybridizing bands. A band approximately $6.8 \mathrm{~kb}$ in size, corresponding to an IS 10 insertion in carFG, was present in all the $\mathrm{Ap}^{\mathrm{R}}$ SBE7 mutants except SBE7 5.5 (Fig. 7), supporting the PCR and DNA sequencing data (Fig. 5). A band at the predicted $7 \cdot 4 \mathrm{~kb}$ was present in the SBE7 5.5 genomic DNA sample, but this co-migrated with at least one other band that was present in SBE7 and all the derived mutant strains. The $7 \cdot 4 \mathrm{~kb}$ band was more distinguishable in SBE7 5.5 than in the other strains when probing for the presence of jemC (data not shown).

There are other examples in the literature of strains that contain multiple copies of IS10: a strain of Enterobacter cloacae has been found to contain at least 15 copies of IS10 (Matsutani, 1991), and a strain of Salmonella enterica has also been shown to possess at least 15 chromosomal copies (Olliver et al., 2005). IS 10 copy number would normally be
Fig. 7. Southern blot to detect the IS 10 transposase gene from chromosomal DNA of several Ecc strains digested with Aatll. The lanes are as follows: A, SBE7 5.1; B, SBE7 5.2; C, SBE7 5.3; D, SBE7 5.4; E, SBE7 5.5; F, SBE7; G, SBE9; H, SBE7 5.6; I, SBE7 5.7; J, SBE7 5.8; K, SBE7 5.9; L, SBE7 5.10. All marker band sizes are approximate values. The dashed arrow shows the predicted $6.8 \mathrm{~kb}$ band that corresponds to an IS10 insertion in carFG in SBE7, which is also indicated with a circle in lane $\mathrm{H}$. Examples of unique IS 10 hybridizing bands present in some of the SBE7 ampicillin-resistant mutants are boxed within rectangles.

kept low by several mechanisms. Firstly the transcription and translation levels of the IS 10R promoter are fairly weak (Simons et al., 1983). Secondly, transcription of the transposase gene is limited to a small fraction of the cell cycle because of methylation of the two dam sites located in IS10R (Roberts et al., 1985). Thirdly, IS10R has been shown to possess mechanisms to limit its copy number in the cell by a process known as 'multicopy inhibition' (Simons \& Kleckner, 1983). This effect is believed to be due to RNA produced by transcription from the $\mathrm{P}_{\text {out }}$ promoter inhibiting translation of the transposase gene by hybridizing to the first $36 \mathrm{bp}$ of the mRNA message and occluding ribosome binding. This effect has been shown to function in trans, so that as the number of IS10 copies in the cell increases, multicopy inhibition becomes more effective at preventing IS 10 transposition. It may be that multicopy inhibition is less effective in some genetic backgrounds, perhaps because the $\mathrm{P}_{\text {out }}$ RNA transcript is turned over rapidly in some strains.

It is possible to differentiate SBE7 5.1-5.10 from each other by the number and size of their IS10R hybridizing bands, which in many cases were different from each other. As an example, SBE7 5.10 contains an easily distinguishable hybridizing band at around $2.2 \mathrm{~kb}$ that is absent in SBE7 and the rest of the $\mathrm{Ap}^{\mathrm{R}}$ SBE7 mutants. Whether these IS10 hybridizing bands were generated before or after selection on Ap is not known. It has been shown that growth in the presence of $\beta$-lactams can induce the SOS response (Miller et al., 2004). The SOS response in turn has been shown to 
induce IS 10 transposition (Eichenbaum \& Livneh, 1998). Therefore, any colonies that are initially able to grow in the presence of Ap may undergo increased rates of IS10R transposition that may explain the high level of heterogeneity among SBE7 5.1-5.10.

One observation from Fig. 7 is that none of the IS10 hybridizing bands present in the SBE7 parental strain is absent in SBE7 5.1-5.10. As IS10R has been shown previously to transpose by a non-replicative mechanism (Bender \& Kleckner, 1986; Kleckner et al., 1995), it seems clear that during transposition, IS10R is maintained in our strains at the donor molecule site. Mechanisms for how this can occur have been described elsewhere (Bender et al., 1991), and our results agree with a model in which intramolecular rejoining to lose IS10R from the donor molecule site is an uncommon event.

\section{Final summary}

This research has generated a functionally expressed $\beta$ lactamase translational fusion in $\mathrm{carH}$, and shown that in the absence of a functional promoter, this construct can serve as an effective mobile promoter trap. This has led to the identification of cryptic IS10 elements in the ATTn10 chromosome. This finding was initially surprising, as it was assumed that IS10 might have been lost from the chromosome during fusaric acid treatment to cure Tn10dependent $\mathrm{Tc}^{\mathrm{R}}$ in the progenitor strain. This promoter trap offered insights into the molecular events that may have led to ATTn10 $\mathrm{Tc}^{\mathrm{S}}$ with the discovery of the Tn10 genetic remnant $\mathrm{Tn} 10 \Delta$ in the SBE7 $\mathrm{Ap}^{\mathrm{R}}$ mutant SBE7 5.5. Southern blot experiments subsequently demonstrated that ATTn10 contains at least 15 copies of IS10. These data show that ATTn10 may be a useful model strain in which to study adaptive evolution, and our recent observations that ATTn10 may be hypermutable are consistent with this notion (S. D. Bowden and G.P.C. Salmond, unpublished data).

\section{ACKNOWLEDGEMENTS}

This work was carried out under DEFRA licence number PHL 177A/ $4995(01 / 2005)$ and funded by a BBSRC research grant. We would also like to thank Mr Ian Foulds for excellent technical support.

\section{REFERENCES}

Albertini, A. M., Hofer, M., Calos, M. P. \& Miller, J. H. (1982). On the formation of spontaneous deletions: the importance of short sequence homologies in the generation of large deletions. Cell 29, 319-328.

Altschul, S. F., Gish, W., Miller, W., Myers, E. W. \& Lipman, D. J. (1990). Basic local alignment search tool. J Mol Biol 215, 403-410.

Bainton, N. J., Stead, P., Chhabra, S. R., Bycroft, B. W., Salmond, G. P. C., Stewart, G. S. A. B. \& Williams, P. (1992). $N-(3-$ oxohexanoyl)-L-homoserine lactone regulates carbapenem antibiotic production in Erwinia carotovora. Biochem J 288, 997-1004.
Balbinder, E. (2001). Stationary phase deletions in Escherichia coli. I - evidence for a new deletion pathway. Mutat Res 479, 19-36.

Bender, J. \& Kleckner, N. (1986). Genetic evidence that $\operatorname{Tn} 10$ transposes by a nonreplicative mechanism. Cell 45, 801-815.

Bender, J., Kuo, J. \& Kleckner, N. (1991). Genetic evidence against intramolecular rejoining of the donor DNA molecule following IS 10 transposition. Genetics 128, 687-694.

Bochner, B. R., Huang, H., Schieven, G. L. \& Ames, B. N. (1980). Positive selection for loss of tetracycline resistance. J Bacteriol 143, 926-933.

Bogosian, G., Bilyeu, K. \& O'Neil, J. P. (1993). Genome rearrangements by residual IS 10 elements in strains of Escherichia coli K-12 which had undergone Tn10 mutagenesis and fusaric acid selection. Gene 133, 17-22.

Chalmers, R. M. \& Kleckner, N. (1996). IS $10 / \operatorname{Tn} 10$ transposition efficiently accommodates diverse transposon configurations. EMBO J 15, 5112-5122.

Chalmers, R., Sewitz, S., Lipkow, K. \& Crellin, P. (2000). Complete nucleotide sequence of Tn10. J Bacteriol 182, 2970-2972.

Ciampi, M. S., Schmid, M. B. \& Roth, J. R. (1982). Transposon Tn 10 provides a promoter for transcription of adjacent sequences. Proc Natl Acad Sci U S A 79, 5016-5020.

Coulthurst, S. J., Barnard, A. M. L. \& Salmond, G. P. C. (2005). Regulation and biosynthesis of carbapenem antibiotics in bacteria. Nat Rev Microbiol 3, 295-306.

Eichenbaum, Z. \& Livneh, Z. (1998). UV light induces IS 10 transposition in Escherichia coli. Genetics 149, 1173-1181.

Farabaugh, P. J., Schmeissner, U., Hofer, M. \& Miller, J. H. (1978). Genetic studies of the lac repressor. VII. On the molecular nature of spontaneous hotspots in the lacI gene of Escherichia coli. J Mol Biol 126, 847-857.

Grinter, N. J. (1983). A broad-host-range cloning vector transposable to various replicons. Gene 21, 133-143.

Halling, S. M. \& Kleckner, N. (1982). A symmetrical six-base-pair target site sequence determines $\mathrm{Tn} 10$ insertion specificity. Cell 28, 155-163.

Herrero, M., De Lorenzo, V. \& Timmis, K. N. (1990). Transposon vectors containing non-antibiotic resistance selection markers for cloning and stable chromosomal insertion of foreign genes in Gramnegative bacteria. J Bacteriol 172, 6557-6567.

Jellen-Ritter, A. S. \& Kern, W. V. (2001). Enhanced expression of the multidrug efflux pumps AcrAB and AcrEF associated with insertion element transposition in Escherichia coli mutants selected with a fluoroquinolone. Antimicrob Agents Chemother 45, 1467-1472.

Kaniga, K., Delor, I. \& Cornelis, G. R. (1991). A wide-host-range suicide vector for improving reverse genetics in Gram-negative bacteria: inactivation of the blaA gene of Yersinia enterocolitica. Gene 109, 137-141.

Kleckner, N., Chalmers, R. M., Kwon, D., Sakai, J. \& Bolland, S. (1995). Tn10 and IS 10 transposition and chromosome rearrangements: mechanism and regulation in vivo and in vitro. Curr Top Microbiol Immunol 204, 49-82.

Kobayashi, K., Tsukagoshi, N. \& Aono, R. (2001). Suppression of hypersensitivity of Escherichia coli acrB mutant to organic solvents by integrational activation of the acrEF operon with the IS1 or IS2 element. J Bacteriol 183, 2646-2653.

Marvo, S. L., King, S. R. \& Jaskunas, S. R. (1983). Role of short regions of homology in intermolecular illegitimate recombination events. Proc Natl Acad Sci U S A 80, 2452-2456. 
Matsutani, S. (1991). Multiple copies of IS10 in the Enterobacter cloacae MD36 chromosome. J Bacteriol 173, 7802-7809.

McGowan, S. J., Sebaihia, M., Porter, L. E., Stewart, G. S. A. B., Williams, P., Bycroft, B. W. \& Salmond, G. P. C. (1996). Analysis of bacterial carbapenem antibiotic production genes reveals a novel $\beta$ lactam biosynthesis pathway. Mol Microbiol 22, 415-426.

McGowan, S. J., Sebaihia, M., O'Leary, S., Hardie, K. R., Williams, P., Stewart, G. S. A. B., Bycroft, B. W. \& Salmond, G. P. C. (1997). Analysis of the carbapenem gene cluster of Erwinia carotovora: definition of the antibiotic biosynthetic genes and evidence for a novel $\beta$-lactam resistance mechanism. Mol Microbiol 26, 545-556.

McGowan, S. J., Barnard, A. M. L., Bosgelmez, G. \& 7 other authors (2005). Carbapenem antibiotic biosynthesis in Erwinia carotovora is regulated by physiological and genetic factors modulating the quorum sensing-dependent control pathway. Mol Microbiol 55, 526-545.

Miller, J. H. (1972). Experiments in Molecular Genetics. Cold Spring Harbor, NY: Cold Spring Harbor Laboratory.

Miller, C., Thomsen, L. E., Gaggero, C., Mosseri, R., Ingmer, H. \& Cohen, S. N. (2004). SOS response induction by $\beta$-lactams and bacterial defense against antibiotic lethality. Science 305, 1629-1631.

Olliver, A., Valle, M., Chaslus-Dancla, E. \& Cloeckaert, A. (2005). Overexpression of the multidrug efflux operon acrEF by insertional activation with IS 1 or IS10 elements in Salmonella enterica serovar Typhimurium DT204 acrB mutants selected with fluoroquinolones. Antimicrob Agents Chemother 49, 289-301.

Prentki, P. \& Krisch, H. M. (1984). In vitro insertional mutagenesis with a selectable DNA fragment. Gene 29, 303-313.
Reading, C. \& Cole, M. (1977). Clavulanic acid: a beta-lactamaseinhibiting beta-lactam from Streptomyces clavuligerus. Antimicrob Agents Chemother 11, 852-857.

Roberts, D., Hoopes, B. C., McClure, W. R. \& Kleckner, N. (1985). IS 10 transposition is regulated by DNA adenine methylation. Cell $\mathbf{4 3}$, 117-130.

Roberts, D. E., Ascherman, D. \& Kleckner, N. (1991). IS 10 promotes adjacent deletions at low frequency. Genetics 128, 37-43.

Sambrook, J., Fritsch, E. F. \& Maniatis, T. (1989). Molecular Cloning: a Laboratory Manual, 2nd edn. Cold Spring Harbor, NY: Cold Spring Harbor Laboratory.

Simons, R. W. \& Kleckner, N. (1983). Translational control of IS10 transposition. Cell 34, 683-691.

Simons, R. W., Hoopes, B. C., McClure, W. R. \& Kleckner, N. (1983). Three promoters near the termini of IS10: pIN, pOUT, and pIII. Cell 34, 673-682.

Singer, B. S. \& Westlye, J. (1988). Deletion formation in bacteriophage T4. J Mol Biol 202, 233-243.

Toth, I., Perombelon, M. C. M. \& Salmond, G. P. C. (1993). Bacteriophage $\phi \mathrm{KP}$ mediated generalised transduction in Erwinia carotovora subsp. carotovora. J Gen Microbiol 139, 2705-2709.

Uematsu, N., Matsuoka, C., Agemizu, Y., Nagoshi, E. \& Yamamoto, K. (1999). Asymmetric crossing over in the spontaneous formation of large deletions in the tonB-trp region of the Escherichia coli K-12 chromosome. Mol Gen Genet 261, 523-529.

Welch, M., Todd, D. E., Whitehead, N. A., McGowan, S. J., Bycroft, B. W. \& Salmond, G. P. C. (2000). N-acyl homoserine lactone binding to the CarR receptor determines quorum-sensing specificity in Erwinia. EMBO J 19, 631-641. 